\title{
Testing for Shock-heated X-Ray Gas around Compact Steep Spectrum Radio Galaxies
}

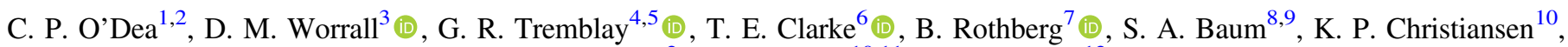 \\ C. A. Mullarkey ${ }^{2}$, J. Noel-Storr ${ }^{10,11}$, and R. Mittal ${ }^{12}$ \\ ${ }^{1}$ Department of Physics \& Astronomy, University of Manitoba, Winnipeg, MB R3T 2N2, Canada; chris.odea@umanitoba.ca \\ ${ }^{2}$ School of Physics \& Astronomy, Rochester Institute of Technology, 84 Lomb Memorial Drive, Rochester, NY 14623, USA \\ ${ }^{3}$ HH Wills Physics Laboratory, University of Bristol, Tyndall Avenue, Bristol BS8 1TL, UK \\ ${ }^{4}$ Einstein Fellow, Department of Astronomy and Yale Center for Astronomy and Astrophysics, Yale University, 52 Hillhouse Avenue, New Haven, CT 06511, USA \\ ${ }^{5}$ Harvard-Smithsonian Center for Astrophysics, 60 Garden Street, Cambridge, MA 02138, USA \\ ${ }^{6}$ Naval Research Laboratory Remote Sensing Division, Code 7213, 4555 Overlook Avenue SW, Washington, DC 20375, USA \\ ${ }^{7}$ Large Binocular Telescope Observatory, 933 North Cherry Avenue, Tucson, AZ 85721, USA \\ ${ }^{8}$ Carlson Center for Imaging Science, Rochester Institute of Technology, 84 Lomb Memorial Drive, Rochester, NY 14623, USA \\ ${ }^{9}$ Faculty of Science, University of Manitoba, Winnipeg, MB R3T 2N2, Canada \\ ${ }^{10}$ InsightSTEM, 855 East River Road \#37, Tucson, AZ 85718, USA \\ ${ }^{11}$ Designated Campus Colleague, Steward Observatory, University of Arizona, 933 North Cherry Avenue, Tucson, AZ 85719, USA \\ ${ }^{12}$ Center for Computational Relativity and Gravitation, Rochester Institute of Technology, 170 Lomb Memorial Drive, Rochester, NY 14623, USA \\ Received 2017 May 10; revised 2017 October 16; accepted 2017 November 6; published 2017 December 15
}

\begin{abstract}
We present Chandra and XMM-Newton X-ray, Very Large Array (VLA) radio, and optical observations of three candidate compact steep spectrum (CSS) radio galaxies. CSS sources are of a galactic scale and are presumably driving a shock through the interstellar medium (ISM) of their host galaxy. B3 1445+410 is a low-excitation emission line CSS radio galaxy with possibly a hybrid Fanaroff-Riley FRI/II (or fat double) radio morphology. The Chandra observations reveal a point-like source that is well fit with a power law consistent with the emission from a Doppler boosted core. 3C 268.3 is a CSS broad-line radio galaxy (BLRG) whose Chandra data are consistent spatially with a point source centered on the nucleus and spectrally with a double power-law model. PKS B1017-325 is a low-excitation emission line radio galaxy with a bent double radio morphology. While from our new spectroscopic redshift, PKS B1017-325 falls outside the formal definition of a CSS, the XMM-Newton observations are consistent with ISM emission with either a contribution from hot shocked gas or non-thermal jet emission. We compile selected radio and X-ray properties of the nine bona fide CSS radio galaxies with X-ray detections so far. We find that two out of the nine show X-ray spectroscopic evidence for hot shocked gas. We note that the counts in the sources are low and that the properties of the two sources with evidence for hot shocked gas are typical of the other CSS radio galaxies. We suggest that hot shocked gas may be typical of CSS radio galaxies due to their propagation through their host galaxies.
\end{abstract}

Key words: galaxies: active - galaxies: individual (PKS B1017-325, B3 1445+410) - galaxies: jets - X-rays: galaxies

\section{Introduction}

The required gaseous medium for the propagation of extragalactic radio sources is provided by the X-ray-emitting atmospheres of their host galaxies and clusters. In radio sources of high power, the energy and momentum fluxes are expected to be sufficient to drive a bow shock at a supersonic speed into the ambient medium, heating the gas as it crosses the shock (e.g., Scheuer 1974; Leahy 1991; Heinz et al. 1998). Evidence for such heating has been claimed around Cygnus A (e.g., Carilli et al. 1994; Smith et al. 2002). In cluster-embedded sources of lower power it is common for the radio lobes to displace X-ray-emitting gas (e.g., Boehringer et al. 1993; Bîrzan et al. 2004; Forman et al. 2007; Wise et al. 2007; Tremblay et al. 2012), with sufficient power to balance radiative cooling of the dense gas (e.g., Dunn et al. 2005; Rafferty et al. 2006; Fabian 2012). However, direct evidence for strong shock heating by radio sources remains relatively scarce. Notable exceptions are the closest radio galaxy, Centaurus A (Kraft et al. 2003; Croston et al. 2009), the cluster radio source 3C444 (Croston et al. 2011), and the intermediate-power radio galaxy PKS B2152-699 (Worrall et al. 2012), where prominent shocks have been studied in detail. There has been less routine success in detecting the anticipated dramatic effects of shocks around high-power sources in more typical atmospheres.

A promising source type for observing shock heating is the non-cluster compact steep spectrum (CSS) population, where the radio structures lie within the volume of the host galaxy (for a review see O'Dea 1998). Here, as for the inner expanding structures of Centaurus A, the ambient gas is the cool galaxy atmosphere, which even when shocked should be accessible to detection in the X-ray band. While CSS sources can show indirect evidence of interaction with the gas in their host galaxies (e.g., Saikia \& Gupta 2003; Orienti 2016), the amount of gas directly involved appears to be relatively small (e.g., Axon et al. 2000; Stockton et al. 2007), and early suggestions that CSS sources are compact because they are "frustrated" by the presence of dense environments are now generally disfavored as compared with arguments that they are compact because they are young (e.g., Fanti et al. 1995). Typical age estimates of less than $10^{6}$ years are based on measurements of the curvature in the radio spectra caused by radiative energy losses of the higher-energy electrons over the lifetime of the sources (e.g., Murgia et al. 1999) or through proper motions of the terminal hot spots of the class of smaller and younger compact symmetric objects (CSOs, a subclass of gigahertz-peaked spectrum (GPS) sources; e.g., Owsianik \& Conway 1998; Peck \& Taylor 2000; 
Taylor et al. 2000; Polatidis 2009; An \& Baan 2012). CSS radio sources are powerful, with statistical arguments made that they decline in luminosity as they age (e.g., Fanti et al. 1995; Readhead et al. 1996). As such they are good candidates for searching for shock heating of surrounding gas at a moderate to high Mach number.

The compact nature of CSS sources, and their smaller cousins, GPS sources, has the disadvantage that the emission might be confused with other components of X-ray emission. Quasars, whose X-ray cores and inner jets are enhanced by relativistic beaming, and where the potential to image shock discontinuities is diminished through the jets lying far from the plane of the sky, are not ideal for such a study. Moreover, it has been argued from polarization and multifrequency variability studies that quasar samples of genuinely young sources can be contaminated by blazars (Cotton et al. 2003; Tornikoski et al. 2009). Where radio galaxies have their cores detected in X-ray and separated from other X-ray components, the nuclear emission is much fainter than in quasars and are correlated with the core radio emission (e.g., Worrall \& Birkinshaw 1994; Evans et al. 2006; and see Worrall 2009 for a review). In CSS radio galaxies the radio core emission is often too weak to be separated from the steep-spectrum lobes, particularly at low radio frequencies where it is self-absorbed, and core X-ray emission may be weak compared with that from the gaseous environment or possibly that associated with the steep-spectrum radio lobes. CSS radio galaxies are therefore good candidates for observing shock heating.

While CSS and GPS quasars and broad-line radio galaxies (BLRG) are detected quite readily in X-rays (e.g., O'Dea 1998; Guainazzi et al. 2004; Worrall et al. 2004; Siemiginowska et al. 2005, 2008), the same is not true for radio galaxies. After unsuccessful attempts to detect them with ROSAT (O'Dea et al. 1996), the first detection of a compact radio galaxy was of the GPS source $\mathrm{B} 1345+125 \quad(z=0.122)$ with ASCA (O'Dea et al. 2000). In addition to B1345+125, we are aware of 32 other GPS radio galaxies having reported detections with XMM-Newton or Chandra (Guainazzi et al. 2006; Vink et al. 2006; Siemiginowska et al. 2008; Tengstrand et al. 2009; Siemiginowska et al. 2016). These GPS sources all have radio sizes less than $1 \mathrm{kpc}$ (most are less than $100 \mathrm{pc}$ ) and, even with Chandra, structures on the scale size of the radio cannot be resolved. Count rates are typically low, and all have been fitted to the simplest model of an absorbed single-component power law. Tengstrand et al. (2009) demonstrates a possible anticorrelation between radio-source size and X-ray intrinsic absorption in GPS galaxies (but see Vink et al. 2006 for a different conclusion), as would be consistent with the presence of an inner absorbing torus as seen in powerful radio galaxies with large-scale structures (e.g., Evans et al. 2006) and X-ray emission that arises further from the nucleus in larger GPS sources. This interpretation is also consistent with the finding that GPS quasars show no intrinsic absorption (Siemiginowska et al. 2008). However, while the location of absorption may be probed, the emission mechanism responsible for the X-rays on various spatial scales is far from certain. Siemiginowska et al. (2016) find that their results do not favor thermal emission from the hot interstellar medium (ISM) for the X-ray continuum emission of the CSO subset of GPS galaxies.

Until relatively recently, there has been less effort to detect CSS radio galaxies in X-rays, despite the fact that their radio structures are on spatial scales resolvable with Chandra. Kunert-Bajraszewska et al. (2014) observed seven CSS sources with low radio power and detected four of them. Three of the low radio power CSS sources have extended X-ray emission, $0810+077,0942+355$, and $1321+045$, which is in a cluster of galaxies (Kunert-Bajraszewska et al. 2013), though all of the four detected sources were fit by an absorbed power law.

The low-redshift CSS radio galaxy 3C305 (Heckman et al. 1982) shows shocked hot gas in the X-rays (Massaro et al. 2009; Hardcastle et al. 2012). The hot gas seems likely to be participating in an outflow driven by the radio source, which includes the cool atomic and warm optical emission line gas (Morganti et al. 2005; Hardcastle et al. 2012; Reynaldi \& Feinstein 2013). In such an outflow scenario, the X-rayemitting phase dominates the energetics of the (known) phases of the gas in the outflow (Hardcastle et al. 2012).

The high throughput of XMM-Newton is useful for the detection of CSS radio galaxies, and we made a good detection of the $z=0.267$ galaxy $3 \mathrm{C} 303.1$ in a $40 \mathrm{ksec}$ observation (O'Dea et al. 2006). In the spectrum we found a thermal component at $k T \sim 0.8 \mathrm{keV}$, which seems likely to be produced by the ISM of the host galaxy, and a second harder component. The lack of a detected radio core suggested to us that it is unlikely that the hard component arises from the nucleus. For the harder component to originate from inverseCompton scattering in the brighter radio lobe, the magnetic field would have to be a factor of about 4 lower than the minimum-energy value. We argued that a plausible hypothesis for the second component is that it is gas that is heated by the bow shock of the expanding radio source. Using an expansion speed of $\sim 6000 \mathrm{~km} \mathrm{~s}^{-1}$ as suggested by lifetime and coolingtime arguments, particularly in the emission line nebula (de Vries et al. 1997; O'Dea et al. 2002), the shock would be very strong (Mach number of 13) and the hot gas should have $k T \sim 43 \mathrm{keV}$, consistent with the observed hard spectral component. 3C 303.1 has subsequently been observed with Chandra for $8 \mathrm{ksec}$ as part of a shallow survey of radio sources. Examination of the archival data (Massaro et al. 2010) shows a detection with only 16 photons, but it is consistent with expectations from our XMM-Newton detection. While this is insufficient to probe the spectral structure, we find that photons are distributed in a more extended fashion than would be the case for a point source (Massaro et al. 2010), supporting our hypothesis that the emission arises from gas rather than being non-thermal in nature.

In this paper we report new X-ray observations of the CSS radio galaxies B3 1445+410 $(z=0.195)$ and 3C $268.3(z=0.3717)$ and the candidate CSS source PKS B1017-325, which is at a redshift of $z=0.318$ (based on new Large Binocular Telescope (LBT) optical spectroscopy, see Section 2.5). We adopt $H_{0}=70 \mathrm{~km} \mathrm{~s}^{-1}$ $\mathrm{Mpc}^{-1}, \Omega_{m_{0}}=0.3, \Omega_{\Lambda_{0}}=0.7 .1$ arcsec corresponds to 3.2 and $4.6 \mathrm{kpc}$ at the distances of B3 1445+410 and PKS B1017-325, respectively.

\section{Observations and Analysis}

\subsection{The Candidate CSS Sources}

The CSS B3 $1445+410$ is part of the B3-Very Large Array (VLA) sample of CSS sources defined by Fanti et al. (2001). The CSS sources are a subset of the B3-VLA sample (Vigotti et al. 1989) and have flux densities at $408 \mathrm{MHz} S_{408}>0.8 \mathrm{Jy}$, 


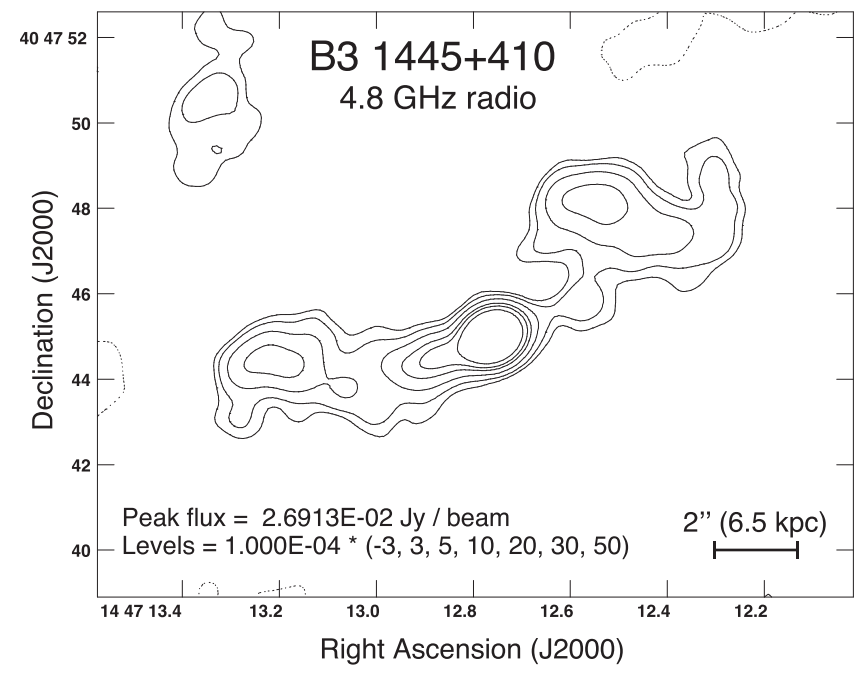

Figure 1. B3 $1445+410$ VLA $4.8 \mathrm{GHz}$ image with UV data tapered to give a resolution of $0.91 \times 0.76$ arcsec at PA $-62^{\circ} .0$ in order to show intermediate scale structure. The radio contours are at $-0.3,0.3,0.5,1.0,2.0,3.0,5.0 \mathrm{mJy}$ beam $^{-1}$.

and based on VLA A configuration $1.5 \mathrm{GHz}$ observations have projected linear diameter $\lesssim 28 \mathrm{kpc}$ and a steep spectral index of $\alpha \lesssim-0.5$ where $S_{\nu} \propto \nu^{\alpha}$. The measured angular size of $\theta \sim 8^{\prime \prime}$ (Figure 1) gives a projected linear diameter of $26 \mathrm{kpc}$.

3C 268.3, where $z=0.3717$, is classified optically as a BLRG. It is a member of the original Fanti et al. (1990) sample of CSS sources. The projected total size of the radio source is $6.9 \mathrm{kpc}$.

The candidate CSS PKS B1017-325 is a member of the Molonglo Southern 4 Jy Sample (MS4; Burgess \& Hunstead 2006a) and was one of 41 sources in MS4 classified as a CSS source by Burgess \& Hunstead (2006b) based on a linear diameter $<25 \mathrm{kpc}$ (using their photometric redshift) and spectral index of $\alpha<-0.5$ between 2700 and $408 \mathrm{MHz}$. Our VLA radio image (Figure 2) shows an angular size of $\theta \sim 9.6 \mathrm{sec}$, which corresponds to a projected linear diameter of $44 \mathrm{kpc}$ using the new spectroscopic redshift and scaling. Thus, PKS B1017-325 is actually larger than the cutoff for CSS sources. We present the data for PKS B1017-325 because it helps provide constraints on the sizes of radio sources that are capable of shocking the ISM. However, we do not include PKS B1017-325 in the analysis of CSS sources in Section 2.7.

\subsection{Chandra}

We observed B3 $1445+410$ at the nominal aimpoint of the S3 CCD of the Chandra ACIS camera on 2009 September 27. The observations were in VFAINT full-frame data mode (OBSID 11579). Results presented here use CIAO v4.8 and CALDB v4.7. We reprocessed the data following the software "threads" from the Chandra X-ray Center $(\mathrm{CXC})^{13}$ to make a new level 2 events file. The observation was free from background flares, and after the removal of time intervals when the background deviated more than $3 \sigma$ from the average value, the total exposure time is $68.541 \mathrm{ksec}$. It was necessary to shift the X-ray coordinates by about 0.26 arcsec, mainly in R.A., to align the nuclear emission with the radio core at R.A. = 14:47:12.758, decl. $=+40: 47: 45.00$. The $0.4-5 \mathrm{keV}$ count rate

\footnotetext{
13 http://cxc.harvard.edu/ciao
}

from a 1.25 arcsec radius circle at the core is 0.01 counts $\mathrm{s}^{-1}$, rendering the pile-up negligible ( $1 \%$ level). The CIAO WAVDETECT task was run to find unassociated point sources that were then excluded from the annular region used to measure the background for our spectral fitting. Spectral fitting was performed using XSPEC version 12.9.0u taking into account the absorption along the line of sight in our Galaxy using a column density of $N_{\mathrm{H}}=1.3 \times 10^{20} \mathrm{~cm}^{-2}$ (from the COLDEN program provided by the CXC, using data of Dickey \& Lockman 1990). A circle of radius 1.25 arcsec was used to extract a binned spectrum of the core.

3C 268.3 was observed with Chandra ACIS-S in VFAINT fullframe data mode on 2009 July 29 and 30 (ObsIDs 10382 and 10933). The initial results were published by Massaro et al. (2015). We reprocessed the data using CIAO 4.9 and CALDB 4.7.5.1, giving a total observing time after background screening of 71.520 ks. Galactic absorption of $N_{\mathrm{H}}=1.91 \times 10^{20} \mathrm{~cm}^{-2}$ was applied to all of the spectral models.

\subsection{XMM-Newton}

We observed PKS B1017-325 with XMM-Newton on 2008 June 11 (ObsID 0552070101). In this paper we report on the data from the European Photon Imaging Camera (EPIC). The pn, MOS1, and MOS2 CCD-array cameras were operated in the full-frame mode with the thin optical blocking filter. The tasks EPCHAIN and EMCHAIN from the SAS were run to create events lists (see http://xmm.esac.esa.int/ for information on the modes of operation of the cameras and analysis procedures). There were no times of extreme background flaring, and the resulting good exposure times, after removing intervals when the background deviated by more than $3 \sigma$ from the average value, were $15.749,15.800$, and $12.832 \mathrm{ksec}$ in the MOS1, MOS2, and pn, respectively. Our analysis uses good events with patterns of $0-12$ from the MOS data and patterns of $0-4$ from the pn data.

For spectral analysis, background-subtracted events were extracted for each camera separately using the task EVSELECT, and corresponding calibration files were made using the RMFGEN and ARFGEN tasks from version 15 of the SAS. Counts were extracted from a source-centered circle, with local background measured from the same CCD. For the pn we followed the recommended procedure of taking background from the same distance to the readout node as the source region. For the MOS cameras this restriction was dropped and somewhat larger background regions were used. The spectral extractions were fitted jointly to models using XSPEC. Data were grouped to 10 counts per bin and the weighting scheme of Churazov et al. (1996) was adopted to provide an improved estimate of the variance in the limit of small numbers of counts. Spectral fits include absorption along the line of sight in our Galaxy assuming a column density of $N_{\mathrm{H}}=5.55 \times 10^{20} \mathrm{~cm}^{-2}$ (from the COLDEN program provided by the CXC using data of Dickey \& Lockman 1990).

\subsection{Radio

$$
\text { 2.4.1. B3 } 1445+410
$$

We obtained archival 4.86 and $8.46 \mathrm{GHz}$ A configuration Karl G. Jansky VLA observations (Program AF0398, 2003 June 01 ) of B3 $1445+410$. The observations were short snapshots with the time on the source of $170 \mathrm{~s}$ at $8.4 \mathrm{GHz}$, and $130 \mathrm{~s}$ at $4.8 \mathrm{GHz}$, respectively. The NRAO AIPS package was 


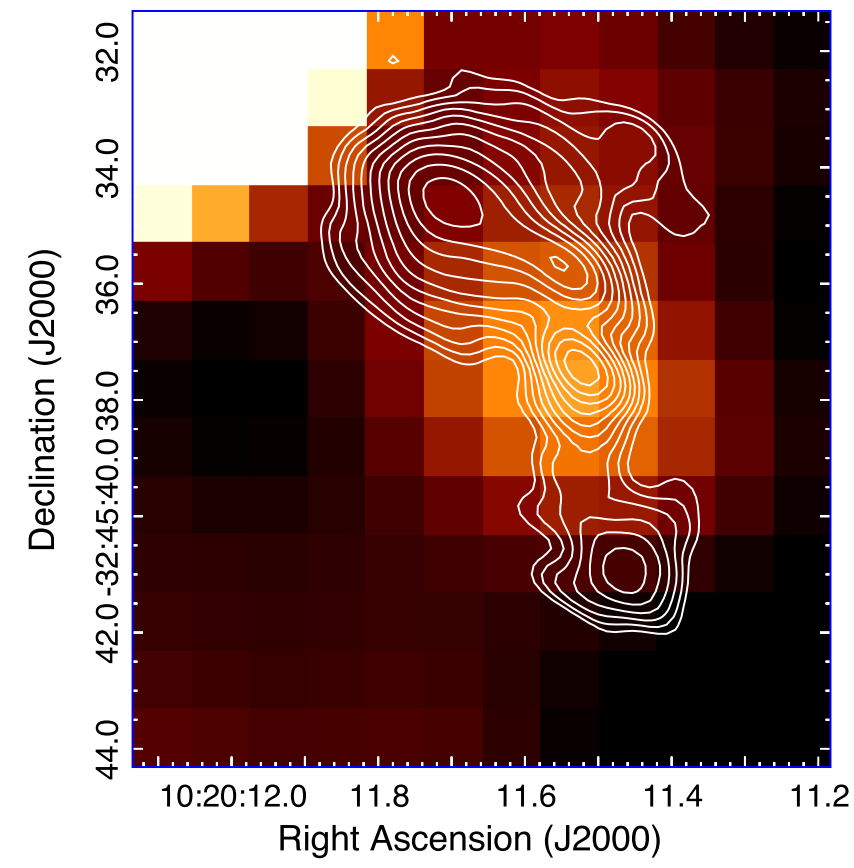

Figure 2. 2MASS (Skrutskie et al. 2006) K-band image of PKS B1017-325 with $22.4 \mathrm{GHz}$ radio contours overlaid. The radio core is cospatial with the host galaxy while a nearby star is seen to the north-east. Contours start at $5 \sigma$ and increase by $\sqrt{2}$, where $\sigma=77.6 \mu \mathrm{Jy} / \mathrm{bm}$.

used for the calibration, imaging, self-calibration, and deconvolution. The $8.46 \mathrm{GHz}$ image (not shown) resolves out nearly all of the extended source structure, however the radio nucleus is detected with a flux density of $S_{8.5} \simeq 25 \mathrm{mJy}$. The core is detected in the $4.86 \mathrm{GHz}$ image with a similar flux density of $S_{4.9} \simeq 26 \mathrm{mJy}$, giving a flat spectral index for the core of $\alpha \simeq-0.03$.

Fanti et al. (2001) present $1.5 \mathrm{GHz}$ VLA A configuration observations of B3 $1445+410$, which show a double morphology. Our tapered $4.86 \mathrm{GHz}$ image (Figure 1) shows the extended structure in more detail. Note that because of the limited $u v$ coverage, structures larger than $\sim 10^{\prime \prime}$ are not adequately imaged. The lobes have weak hot spots and there is a one-sided FRI-like jet extending from the core into the eastern lobe. This structure suggests an intermediate FRI/FRII morphology consistent with the class of "fat doubles" described by Owen \& Laing (1989). The very weak hot spots and plumelike lobes suggest that the lobes are not expanding rapidly into the ISM/ICM.

\subsubsection{PKS B1017-325}

We observed PKS B1017-325 with the NRAO VLA on 2011 February 01 using the hybrid $\mathrm{CnB}$ array with an extended north arm due to the southern declination of the target (Program 10C-233). The observations covered a total block of $1.5 \mathrm{hr}$ with antenna pointing corrections made at $8.44 \mathrm{GHz}$ and the target observed with a total bandwidth of $256 \mathrm{MHz}$ centered on $22.4 \mathrm{GHz}$. The phase calibrator used was J1018-3123 and the flux/bandpass calibrator was 3C 286.

The data were calibrated and imaged with the NRAO Obit package (Cotton 2008) following standard procedures. Briefly, the data were Hanning smoothed to suppress ringing due to radio frequency interference (RFI). They were flagged for RFI that was impulsive in time or frequency. The calibrator 3C 286 was used to determine corrections for instrumental group delay offsets that were applied to all data. 3C 286 was also used to determine the bandpass to correct for residual variations of gain and/or phase with frequency across the band. The flux scale was set using 3C 286 and was bootstrapped to observations of the phase calibrator J1018-3123, which bracketed the target observations. The phase calibrator was used to calibrate the amplitudes and phases of our target. Following this initial full calibration and flagging, we reset the calibration and undertook a second full round of RFI flagging and calibration on only the data that passed the initial calibration round. Calibration was applied to PKS B1017-325 and it was split off for imaging. The data were imaged and underwent three rounds of phaseonly self-calibration. The final image has an rms of $77.6 \mu \mathrm{Jy}$ per beam with a beam of $1.1 \times 00^{\prime \prime} 6$ at a position angle of $48^{\circ} .4$.

The $22.4 \mathrm{GHz}$ image (Figure 2) shows an amorphous morphology with a central core component that is cospatial with an optical and IR point source detected in ground-based imaging. A single Gaussian component fit to the apparent core gives a position of R.A. $102011.52411 \pm 0.0002879$, decl. $-324537.4292 \pm 0.004541$, where the uncertainty in R.A. and decl. is given in arcsec. To the north of the core there is a bright elongated region roughly $4.9 \operatorname{arcsec}(22.5 \mathrm{kpc})$ in length elongated to the north-east. In addition, there is a faint suggestion of further emission extending $\sim 7 \mathrm{kpc}$ to the west of the northern portion of the bright emission. To the south of the core, there is faint emission connecting to a brighter compact region. This emission extends $21.6 \mathrm{kpc}$ to the south of the core. The overall radio source morphology is unclear but might be a bent double or wide-angle-tail radio source (e.g., Owen \& Rudnick 1976; Blanton et al. 2001). The total flux density of all of the components of PKS B1017-325 at $22.4 \mathrm{GHz}$ is $110.0 \pm 0.6 \mathrm{mJy}$ with $14.2 \pm 0.1 \mathrm{mJy}$ in the core component. We note that Gaussian component fitting to the core region reveals an extended structure so we are likely measuring a combination of emission from the central AGN and the surrounding region.

\subsection{Optical Spectroscopy}

$$
\text { 2.5.1. B3 } 1445+410
$$

The Sloan Digital Sky Survey (SDSS) spectrum of B3 1445 +410 is shown in Figure 3. The SDSS observations were taken from plate 1397 and fiber 182. The data sets were used as delivered from the SDSS archive, with no post-processing applied. The spectrum is that of a red elliptical galaxy with low-ionization emission lines, e.g., $\mathrm{H} \alpha+[\mathrm{N}$ II], [O II $] \lambda 3727$, and $\mathrm{H} \beta$. $\mathrm{Mg}$ and $\mathrm{Na}$ are seen in absorption. The redshift is $z=0.1952 \pm 0.0002$. The excitation index $\mathrm{EI}^{14}$ (Buttiglione et al. 2010) is EI $\sim 0.6$, consistent with B3 $1445+410$ being a low-excitation galaxy (LEG, also as defined by Buttiglione et al. 2010).

\subsubsection{LBT Optical Spectroscopy of PKS B1017-325}

An optical spectrum of PKS B1017-325 (Figure 4) was obtained on UT 2017 January 11 using the Multi-Object Double Spectrograph 2 (MODS-2; Pogge et al. 2006). MODS-2 is one of two identical optical low-medium resolution two channel (blue and red) spectrographs mounted on the LBT. MODS-1

\footnotetext{
${ }^{14}$ Buttiglione et al. (2010) defines the excitation index as $\mathrm{EI}=\log [\mathrm{O} \mathrm{III}] / \mathrm{H} \beta$ $-1 / 3(\log [\mathrm{N}$ II $] / \mathrm{H} \alpha+\log [\mathrm{S}$ II $] / \mathrm{H} \alpha+\log [\mathrm{O} \mathrm{I}] / \mathrm{H} \alpha)$.
} 


\section{B3 1445+410 SDSS Spectrum}

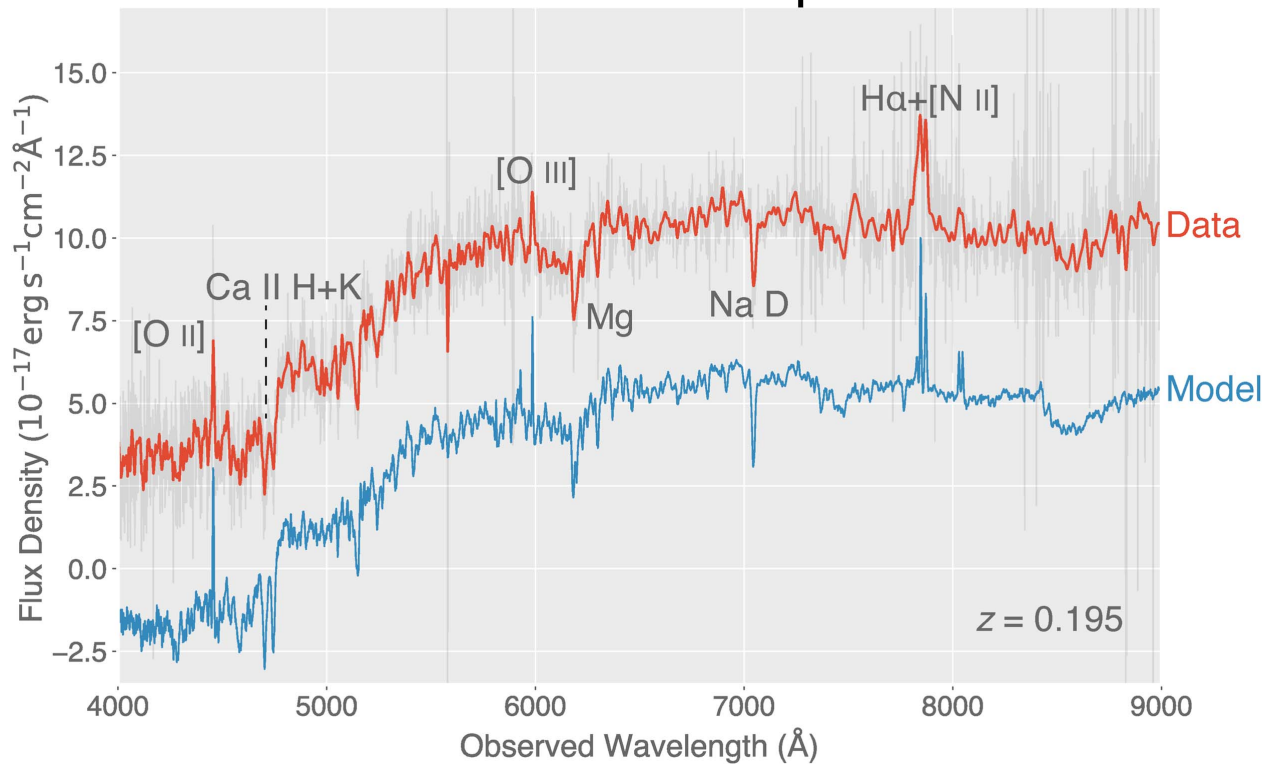

Figure 3. B3 1445+410 SDSS spectrum showing a red elliptical galaxy with low-ionization emission lines.

1e-15 PKS B1017-325 LBT Spectrum

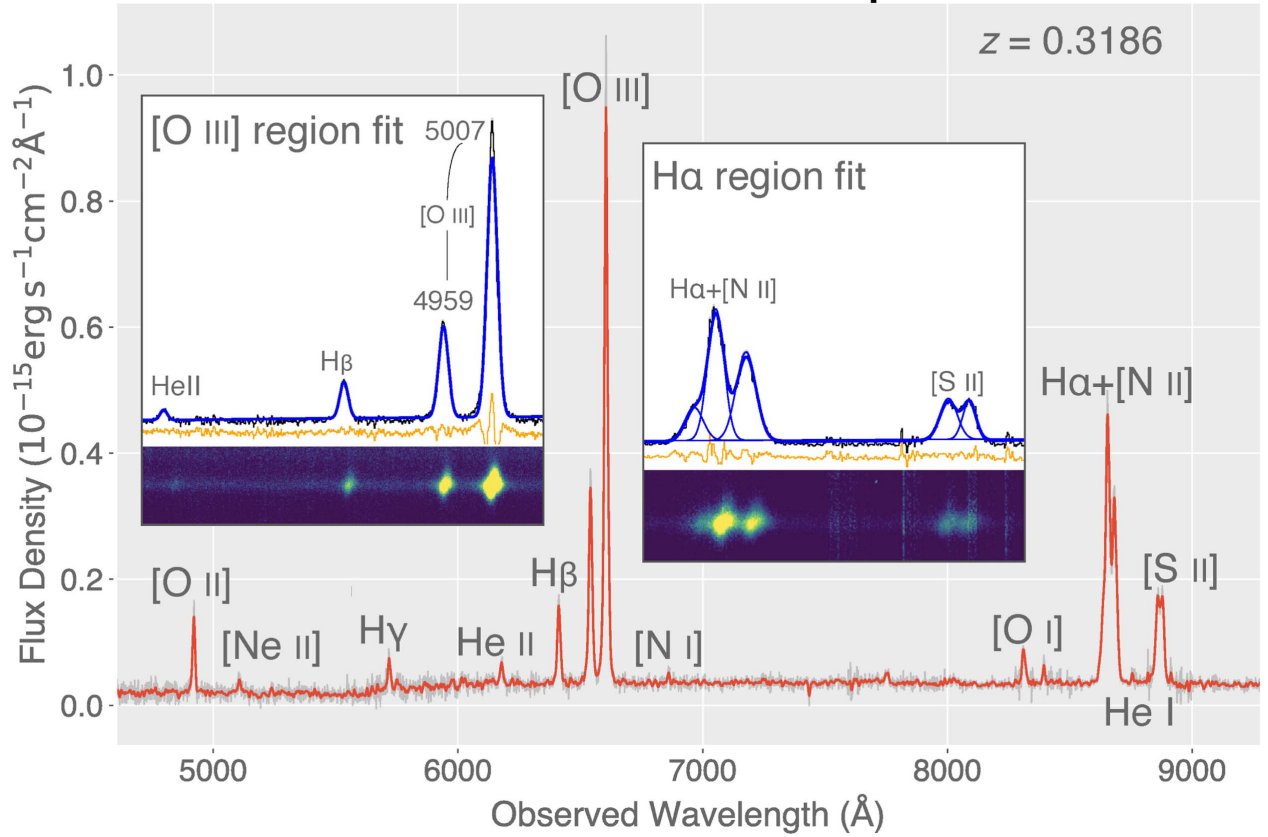

Figure 4. The new LBT/MODS-2 optical spectrum of PKS B1017-325. The inset panels show the results of simultaneous multi-component Gaussian fits to emission lines in the [O III] and $\mathrm{H} \alpha$ regions of the spectrum. In those inset panels, the data and error bars are shown in black and gray (respectively), our composite multicomponent fit to the data is shown in blue, and the residual between that fit and the data is shown in orange. All of the detected lines in the spectrum are consistent with a redshift of $z=0.3186 \pm 0.009$. This, the first spectroscopic redshift yet obtained for PKS B1017-325, is inconsistent with the photometric redshift reported for the source in the NASA/IPAC Extragalactic Database (NED).

and MODS- 2 are each mounted on one of the two $f / 15$ straight Gregorian foci of the two $11.8 \mathrm{~m}$ mirrors. MODS-2 is located on the DX (or right) side of the telescope. Although the LBT has moved to nearly full-time binocular duplex (identical configuration) observations with all three of its facility instruments, including MODS-1 and 2 (Rothberg et al. 2016), PKS 1017-325 could only be observed in monocular mode primarily due to the low declination of the target.
In standard Gregorian or non-prime focus operations, LBT uses an adaptive secondary (AdSec) mirror with each primary mirror (Gallieni et al. 2003; Quirós-Pacheco et al. 2010; Miller et al. 2012). Each AdSec employs a thin shell $91.1 \mathrm{~cm}$ mirror with 672 actuators. These mirrors are limited in operation to elevations above $30^{\circ}$ even when used in seeing limited (nonadaptive) mode. LBT has a single rigid secondary mirror that is not subject to the same limits. As PKS 1017-325 never 
reaches an elevation above $30^{\circ}$ at Mount Graham, only the rigid secondary, which was already mounted on the DX side of the telescope, could be used.

Either MODS spectrograph can be figured to simultaneously observe the wavelength range of $0.3-1.0 \mu \mathrm{m}$ using a dichroic to split the light into a blue and red channel. MODS-2 was configured in the dual grating mode using a 1 !' 2 wide segmented longslit mask. The mask contains five longslit segments, each $60^{\prime \prime}$ in length. A single segment was sufficient for the observations. The spatial scale of the blue arm and red arms are 0 "' 120 pixel $^{-1}$ and 0 !' 123 pixel $^{-1}$, respectively. The observations were obtained under nearly full Moon (98.7\%) conditions with consistent thick clouds and seeing of $\sim 2^{\prime \prime}$, as determined from the acquisition image. A single blue and red exposure of $1200 \mathrm{~s}$ was taken of PKS 1017-325. The spectrophotometric standard G191B2b was observed using the same instrumental setup as the science observations, with the exception of the slitwidth. A $5^{\prime \prime}$ wide slit was used in order to minimize light loss for flux calibrations. Slitless pixel flats were obtained to correct for the detector response. Ar, $\mathrm{Xe}, \mathrm{Kr}$, $\mathrm{Ne}$, and $\mathrm{Hg}$ lamps were observed for wavelength calibration.

The first step of the data reduction was to use Version 2.0 of the modsCCDRed collection of Python scripts. These perform basic $2 \mathrm{D}$ reduction including bias subtraction, removing the overscan regions, constructing flat fields from the slitless pixel flats, fixing bad columns, and flipping the red arm data so that wavelength increases from left to right along the $x$-axis. Further processing was performed using customized IRAF scripts developed by B. Rothberg (Secrest et al. 2015). Briefly, cosmic rays were removed using the task CRUTIL. Two-dimensional spectra were extracted in strip mode for the central longslit segment using the task APALL. MODS spectra are tilted along both the spectral ( $x$-axis) and spatial (y-axis) dimensions. The spectra were simultaneously corrected in both axes using the arc lines and the IRAF tasks ID, REID, FITC, and TRANSFORM. MODS data are very sensitive to the polynomial order used to wavelength calibrate the data. A 4th order Legendre polynomial produces the smallest residuals and avoids introducing low-order noise into the 2D spectra. Once the 2D spectra were rectified and wavelength calibrated, the background was subtracted in each exposure by fitting a 2 nd order Legendre polynomial to the columns (spatial axis) using the IRAF task BACKGROUND. A 1D spectra was extracted in a metric aperture of $2^{\prime \prime}$ for both the blue and red exposures. The exposures were then flux calibrated using the spectrophotometric standard G1919B2b. This step also removes the instrumental signature. The red data $(0.56-1.0 \mu \mathrm{m})$ were corrected for telluric absorption features using a normalized spectra of G191B2b in conjunction with the IRAF task TELLURIC. The data were then corrected for atmospheric extinction and Galactic reddening assuming $R_{\mathrm{V}}=3.1$ and a value of $A_{\mathrm{V}}=0.178$ (Schlafly \& Finkbeiner 2011). Finally, the 1D blue and red spectra were combined using the IRAF task SCOMBINE, corrected to a heliocentric velocity, rescaled to a common dispersion value of $0.85 \AA \mathrm{pixel}^{-1}$, and trimmed to a wavelength range of $0.38-0.9285 \mu \mathrm{m}$. Measurements of the sky lines yield a spectral resolution of $4.2 \AA$ in the blue and $7.3 \AA$ in the red, which corresponds to a resolution of $R$ $\sim 1300$ across the entire spectral range.

Simultaneous Gaussian fits to the [O III] $\lambda \lambda 4959,5007$, [O I] $\lambda 6300$, and [S II] $\lambda \lambda 6718,6732$ emission lines give a spectroscopic redshift of $z_{\text {spec }}=0.318 \pm 0.009$, with which all
Table 1

Emission Line Properties of PKS B1017-325

\begin{tabular}{|c|c|c|}
\hline Line & $\begin{array}{l}\text { FWHM } \\
\left(\mathrm{km} \mathrm{s}^{-1}\right)\end{array}$ & $\begin{array}{c}\text { Flux } \\
\left(\times 10^{-15} \mathrm{erg} \mathrm{s}^{-1} \mathrm{~cm}^{-2}\right)\end{array}$ \\
\hline$[\mathrm{O}$ II $] \lambda 3726 \AA$ & $627 \pm 94$ & $1.54 \pm 0.23$ \\
\hline$[\mathrm{Ne}$ III] $\lambda 3868 \AA$ & $573 \pm 85$ & $0.214 \pm 0.03$ \\
\hline $\mathrm{H} \gamma \lambda 4340 \AA$ & $539 \pm 80$ & $0.576 \pm 0.09$ \\
\hline He II $\lambda 4685 \AA$ & $551 \pm 83$ & $0.427 \pm 0.06$ \\
\hline $\mathrm{H} \beta \lambda 4861 \AA$ & $656 \pm 98$ & $1.91 \pm 0.18$ \\
\hline$[\mathrm{O}$ III $] \lambda 4959 \AA$ & $660 \pm 99$ & $4.87 \pm 0.73$ \\
\hline$[\mathrm{O} \mathrm{III}] \lambda 5007 \AA$ & $665 \pm 98$ & $14.8 \pm 2.2$ \\
\hline$[\mathrm{N}$ I] $\lambda 5198 \AA$ & $337 \pm 51$ & $0.162 \pm 0.02$ \\
\hline$[\mathrm{O}$ I $] \lambda 6300 \AA$ & $491 \pm 74$ & $0.852 \pm 0.13$ \\
\hline [O I] $\lambda 6363 \AA$ & $302 \pm 45$ & $0.285 \pm 0.04$ \\
\hline$[\mathrm{N}$ II $] \lambda 6548 \AA$ & $658 \pm 99$ & $1.99 \pm 0.30$ \\
\hline $\mathrm{H} \alpha \lambda 6563 \AA$ & $602 \pm 90$ & $8.11 \pm 1.2$ \\
\hline$[\mathrm{N}$ II $] \lambda 6583 \AA$ & $736 \pm 111$ & $6.50 \pm 0.98$ \\
\hline [S II] $\lambda 6716 \AA$ & $418 \pm 63$ & $2.78 \pm 0.42$ \\
\hline [S II $] \lambda 6731 \AA$ & $418 \pm 63$ & $2.06 \pm 0.31$ \\
\hline
\end{tabular}

Note. Emission line properties from the new LBT optical spectrum acquired for PKS B1017-325. Only those lines detected at $\gtrsim 5 \sigma$ are reported. The redshift of all of the detected lines reported above is $z=0.318 \pm 0.009$. The excitation index as defined by Buttiglione et al. (2010) is EI $\sim 0.63$, consistent with the source being a LEG.

of the other detected lines are consistent. This is the first and only known spectroscopic redshift for PKS B1017-325, and we note that it is inconsistent with the photometric redshift of $z_{\text {phot }} \approx 0.17$ as reported in Burgess \& Hunstead (2006b) and quoted by the NED. The measured Balmer decrement $(\mathrm{H} \alpha / \mathrm{H} \beta$ flux ratio) is $R_{\mathrm{obs}} \approx 4.42$. Following Tremblay et al. (2010), this corresponds to a color excess $E(B-V)$ of

$$
E(B-V)_{\mathrm{H} \alpha / \mathrm{H} \beta}=\frac{2.5 \times \log \left(2.86 / R_{\mathrm{obs}}\right)}{k\left(\lambda_{\alpha}\right)-k\left(\lambda_{\beta}\right)}=0.437,
$$

where $k\left(\lambda_{\alpha}\right) \approx 2.63$ and $k\left(\lambda_{\beta}\right) \approx 3.71$ as given by Cardelli et al. (1989). The excitation index as defined by Buttiglione et al. (2010) is EI $\sim 0.63$, consistent with the source being a LEG. Typical linewidths (e.g., for [O III] and $\mathrm{H} \alpha$ ) are on the order of $\sim 600 \mathrm{~km} \mathrm{~s}^{-1}$. Fluxes and velocity widths of all $\gtrsim 5 \sigma$ detected emission lines are given in Table 1.

\subsection{X-Ray Imaging and Spectroscopy}

Note that because B3 1445+410 and PKS B1017-325 are optically classified as LEGs, it is unlikely that there is detectable X-ray emission from an accretion disk in these sources (e.g., Hardcastle et al. 2009).

\subsection{1. $B 31445+410$}

Figure 5 (left) is the Chandra image with radio contours and shows the X-ray emission to be concentrated around the radio core with no obvious emission from (or structure induced by) the extended radio features. To test for spatial extent we modeled the X-ray point-spread function (PSF) appropriate for the observation using the CXC SAOsac raytrace and MARX software. Fifty individual simulations were added and the result was compared with the data. Figure 5 (right) shows the X-ray data after using the CIAO ARESTORE task to remove the PSF 


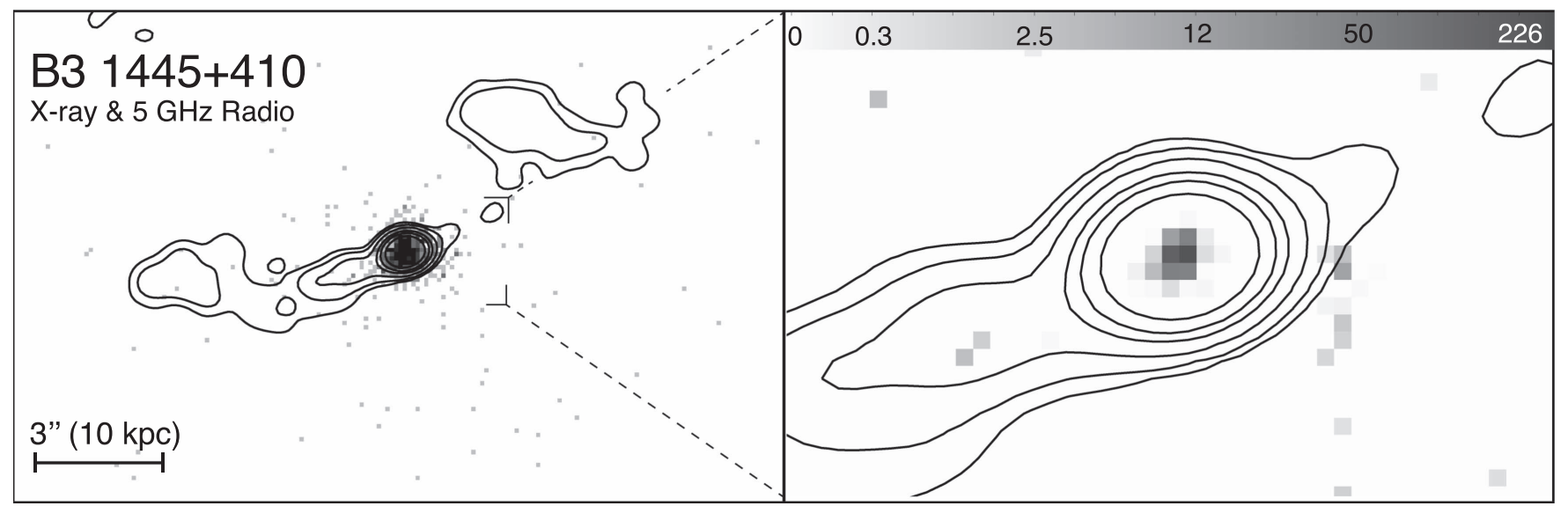

Figure 5. (Left) Chandra $0.4-5 \mathrm{keV}$ data for $\mathrm{B} 31445+410$ with $5 \mathrm{GHz}$ radio contours. The Chandra data are subsampled into pixels of 0.0984 arcsec $(1 / 5$ of the native detector pixel size). The radio contours are at $0.3,0.5,1,2,3,5 \mathrm{mJy}$ beam ${ }^{-1}$ and the restoring beam is $0{ }^{\prime \prime} 57 \times 00^{\prime \prime} 37$. The core flux density is roughly $26 \mathrm{mJy}$ at $5 \mathrm{GHz}$. (Right) Same as for the left panel, with a zoom into the central region, where the X-ray data have been deconvolved with the Chandra PSF. The scale is in units of X-ray counts.

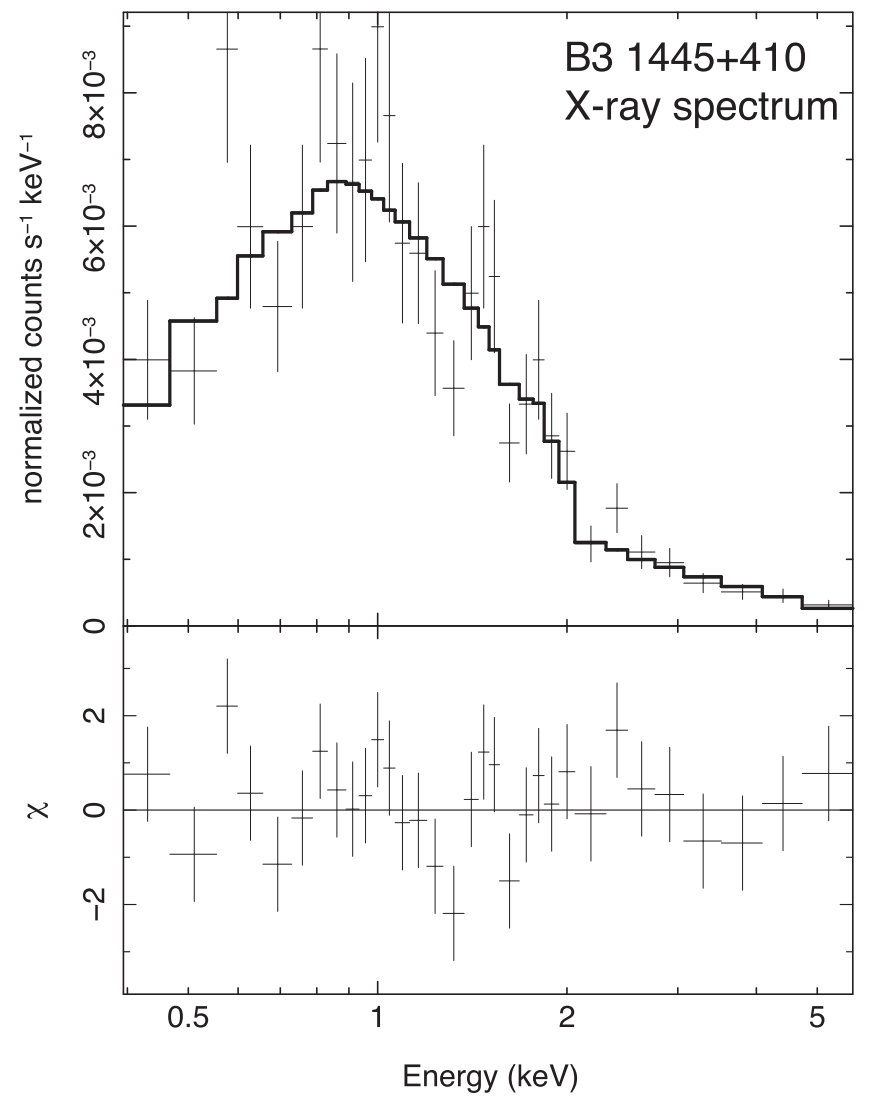

Figure 6. Chandra core spectrum of B $1445+410$ fitted to a power-law model. The lower panel shows residuals expressed as their individual contributions, $\chi$, to $\chi^{2}$.

blurring using the modeled PSF and the Richardson Lucy deconvolution algorithm. A point source is recovered.

The X-ray spectrum (Figure 6) gives a good fit $\left(\chi^{2}=29.6\right.$ for 30 degrees of freedom) to a power law of X-ray spectral index $\Gamma=1.67 \pm 0.11$ (90\% confidence), where $\Gamma=\alpha+1$ and the flux density is given by $S \propto \nu^{-\alpha}$. No absorption in excess of the Galactic is required. The $1 \mathrm{keV}$ flux density is $8.9 \pm 0.6 \mathrm{nJy}$, and between 0.4 and $6 \mathrm{keV}$ the observed flux is $(6.8 \pm 0.5) \times 10^{-14} \mathrm{erg} \mathrm{cm}^{-2} \mathrm{~s}^{-1}$ and the luminosity is
$(7.2 \pm 0.5) \times 10^{42} \mathrm{erg} \mathrm{s}^{-1}$ (90\% confidence uncertainties for one interesting parameter).

The observation that the radio core is a flat spectrum and relatively bright and that there is a one-sided radio jet is consistent with there being some relativistic boosting. Thus, the measurement that the $\mathrm{X}$-rays are point-like and consistent with a power-law spectrum suggests that the X-ray emission is nonthermal emission from the radio core and/or base of the jet.

\subsection{2. $3 C 268.3$}

The X-ray emission appears unresolved on inspection. We confirmed this by simulating a PSF using the CXC SAOsac RAYTRACE and MARX software, and using the CIAO ARESTORE task to apply the Richardson Lucy deconvolution algorithm, as was used to show that the CSS source B3 1445 +410 is also compact in X-rays. The model PSF was constructed using the spectral distribution of counts found in the data (see below). The X-ray results before and after deconvolution are shown in Figure 7, along with contours from a $5 \mathrm{GHz}$ MERLIN radio map of the source. A small astrometric correction of 0.17 arcsec was applied to the X-ray data to center the source at 12:06:24.715, 64:13:36.98, which is the unconfirmed position of the radio core given by Lüdke et al. (1998).

The spectrum was measured from counts between energies of 0.4 and $7 \mathrm{keV}$ extracted from a circle with a radius of 1.25 arcsec, with background from an annulus of radii 5 and 90 arcsec, masking sources in the background region found by the CIAO wavdetect software. Counts were grouped to 15 per spectral bin, and the weighting scheme of Churazov et al. (1996) was adopted to provide an improved estimate of the variance in the limit of small numbers of counts. A singlecomponent absorbed power-law model indicated high absorption but gives an unacceptable fit $\left(\chi^{2}=71.1\right.$ for 37 degrees of freedom; dof). An acceptable fit $\left(\chi^{2}=31.8\right.$ for 35 dof $)$ is found when a second, less absorbed, power law is added (Figure 8). The slope of the second power law is poorly constrained and so we have fixed it at $\alpha_{2}=0.7$. The component values, where $S$ is the unabsorbed $1 \mathrm{keV}$ flux density and $L$ is the unabsorbed $0.4-7 \mathrm{keV}$ luminosity, with 



Figure 7. 3C 268.3. Chandra 0.5-5 keV data, with green contours at $0.4,1,4,10,40,100 \mathrm{mJy}$ per beam from the MERLIN $5 \mathrm{GHz}$ map of Lüdke et al. (1998) downloaded from the NED. The Chandra data are subsampled into pixels of $0.0984 \operatorname{arcsec}(1 / 5$ of the native detector pixel size). Left: Chandra image. Right: X-ray data after deconvolution with the Chandra PSF. Scales are in X-ray counts.

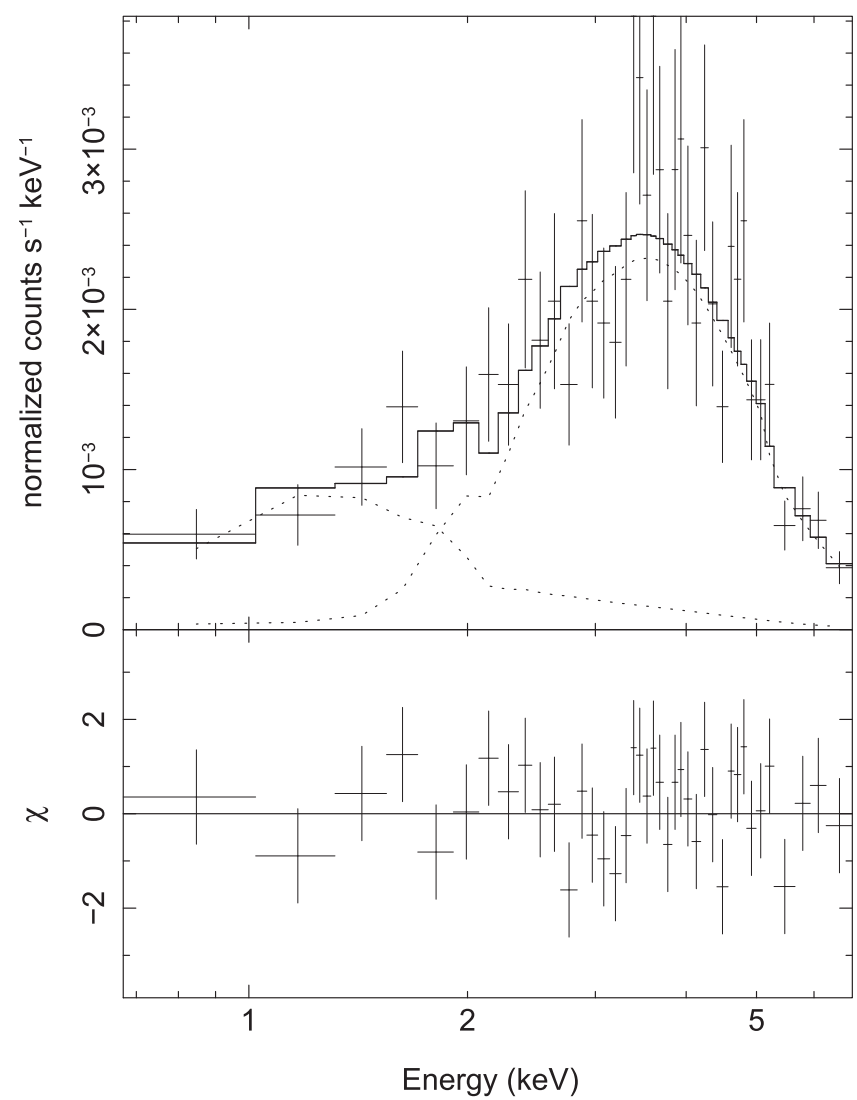

Figure 8. 3C 268.3. Chandra spectrum fit to two absorbed power laws as described in the text.

$90 \%$ confidence errors for 1 interesting parameter, are as follows.

$$
\begin{aligned}
& \alpha_{1}=0.85_{-0.5}^{+0.6}, N_{\mathrm{H}-1}=1.5_{-0.4}^{+0.6} \times 10^{23} \mathrm{~cm}^{-2}, S_{1}=83_{-48}^{+153} \mathrm{nJy}, \\
& \text { and } L_{1}=2.9 \times 10^{44} \mathrm{erg} \mathrm{s}^{-1} . \\
& \alpha_{2}=0.7 \text { (fixed), } N_{\mathrm{H}-2}=6.6_{-5.7}^{+8.3} \times 10^{21} \mathrm{~cm}^{-2} S_{2}=2.4_{-1.2}^{+2.1} \mathrm{nJy},
\end{aligned}
$$$$
L_{2}=8.8 \times 10^{42} \mathrm{erg} \mathrm{s}^{-1} \text {. }
$$

Merely adding a soft thermal component to the heavily absorbed power law is not acceptable (the temperature pegs at the upper limit of $64 \mathrm{keV}$ ), but we cannot rule out an (expected) small contribution to the spectrum from 3C 268.3's ISM.

\subsubsection{PKS B1017-325}

$\mathrm{X}$-ray emission is seen in each of the three XMM-Newton EPIC cameras, centered within 1.5 arcsec of the location of PKS B1017-325. Figure 9 shows the X-ray image with radio contours. Although the X-ray source appears to be centered slightly to the southeast of the radio nucleus, this is within $X M M$-Newton's astrometric uncertainties of a few arcseconds, as confirmed by Watson et al. (2009) during the creation of the 2XMM source catalog. The X-ray source is compact, essentially lying within the circle with a radius of $12^{\prime \prime}$ we used for spectral extractions. The data show no excess centered on the location of foreground star (U0525_12970154 in the USNO-A2.0 catalog) with $B=11.9 \mathrm{mag}$ and $r=13.8 \mathrm{mag}$ (marked by the small circle in Figure 9), implying an X-ray to optical flux ratio less than about $3 \times 10^{-5}$, consistent with findings for stars that are this blue in color (e.g., Vaiana et al. 1981).

The X-ray spectrum (Figure 10) gives a good fit $\left(\chi^{2}=13.0\right.$ for $13 \mathrm{dof}$ ) to a power law of spectral index of $\Gamma=2.83 \pm 0.36$ ( $90 \%$ confidence), where $\Gamma=\alpha+1$ and the flux density is given by $S \propto \nu^{-\alpha}$. The $1 \mathrm{keV}$ flux density is $2.5 \pm 0.6 \mathrm{nJy}$, and between 0.4 and $6 \mathrm{keV}$ the observed flux is $(1.1 \pm 0.2) \times 10^{-14}$ $\mathrm{erg} \mathrm{cm} \mathrm{cs}^{-2} \mathrm{~s}^{-1}$ and the luminosity is $(5.8 \pm 1.1) \times 10^{42} \mathrm{erg} \mathrm{s}^{-1}$ (90\% confidence uncertainties for one interesting parameter).

Since the spectral slope is unusually steep for a radio-galaxy core (e.g., Evans et al. 2006; Massaro et al. 2010, 2012, 2013), and the XMM-Newton PSF is large enough to contain a significant galaxy atmosphere, we have also explored fits to a thermal (APEC) model. We fixed the abundances to Solar, since values close to that are confirmed observationally for early-type galaxies (e.g., Konami et al. 2014) and there is no evidence in luminosity or extent for a significant outer atmosphere where lower abundances are typical. A single-component thermal gives a poor fit $\left(\chi^{2}=25.1\right.$ for 13 dof $)$, and adding a thermal to the power-law model finds essentially the power-law component alone as it pushes $k T$ to its maximum bound of $64 \mathrm{keV}$. When we fix the power-law index to $\Gamma=1.7$, the value for B3 $1445+410$, 


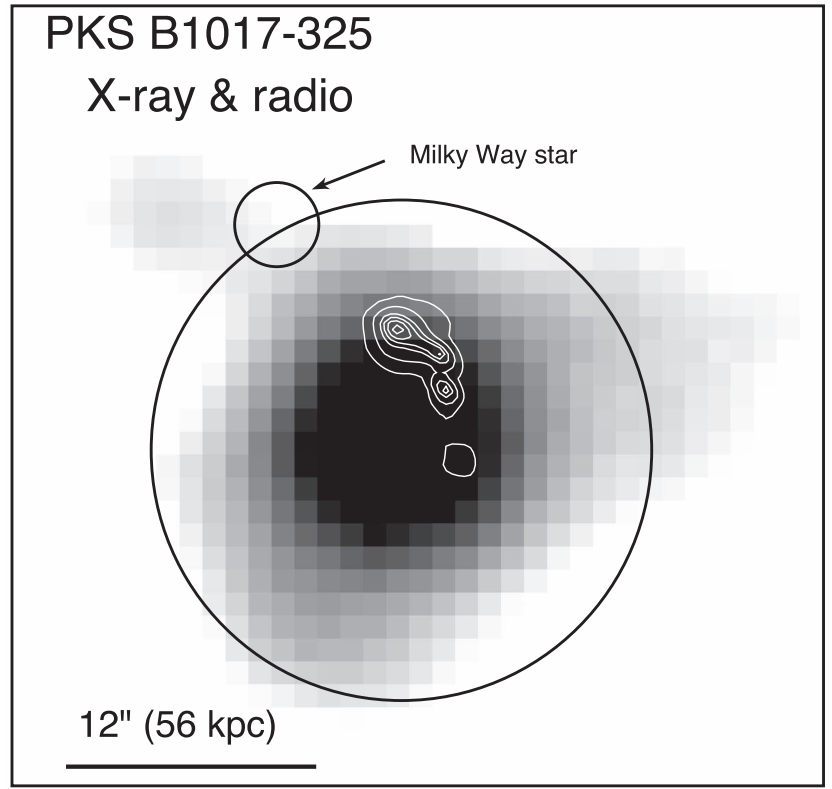

Figure 9. 0.4-5 keV XMM-Newton image of PKS B1017-325 in 1 arcsec bins and smoothed with a Gaussian of $\sigma=3^{\prime \prime}$. The large circle has a radius of $12^{\prime \prime}$ and the small circle marks the location of USNO star U0525_12970154. The white radio contours from our $22.5 \mathrm{GHz}$ map are at $1,3,6,8,10,12 \mathrm{mJy}^{-1} \mathrm{beam}^{-1}$ and the map has a restoring beam of $1.1 \times 0.6$ arcsec. The core flux density is roughly $9 \mathrm{mJy}$ at $22.5 \mathrm{GHz}$

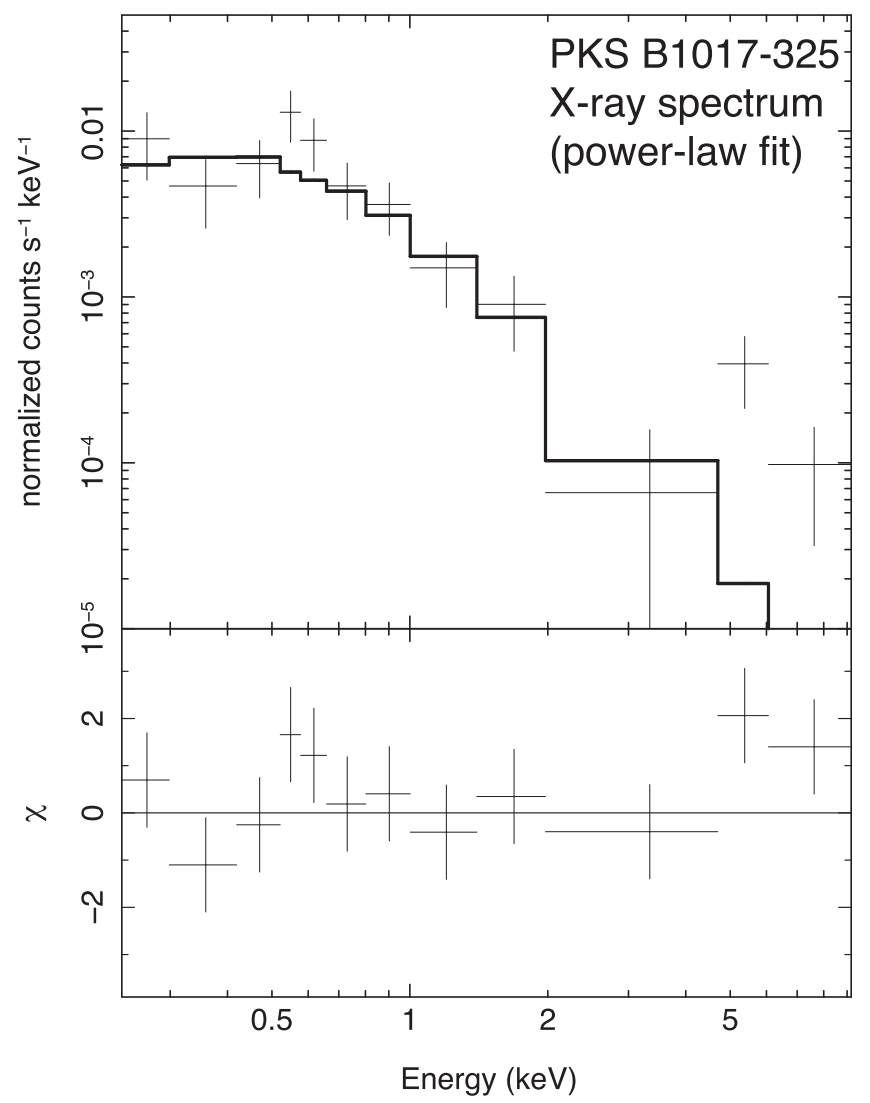

Figure 10. XMM-Newton core spectrum of PKS B1017-325 fitted to a power-law model. The lower panel shows residuals expressed as their individual contributions, $\chi$, to $\chi^{2}$. For clarity of display only the pn data are shown.

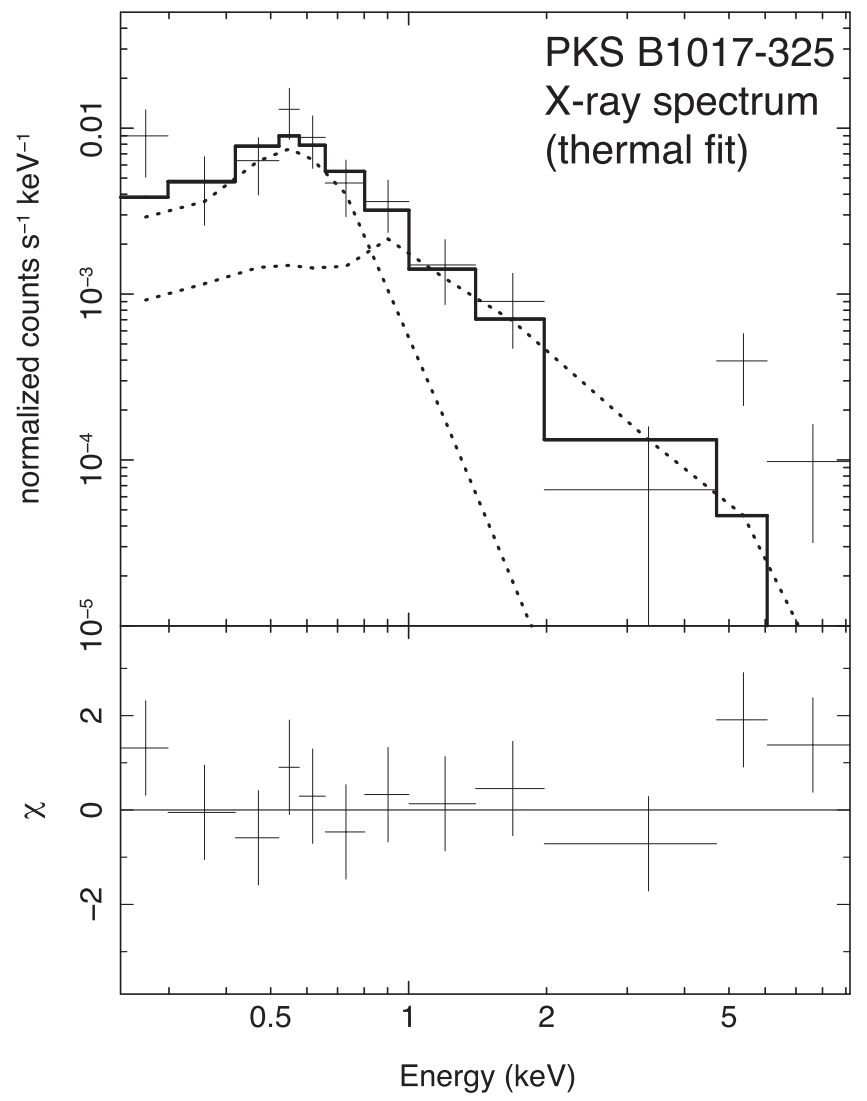

Figure 11. XMM-Newton core spectrum of PKS B1017-325 fitted to two thermal models. The dotted lines show the individual model contributions to the fit. The lower panel shows residuals expressed as their individual contributions, $\chi$, to $\chi^{2}$. For clarity of display only the pn data are shown.

the best-measured X-ray spectral index of any CSS radio galaxy dominated by core emission, the two-component model finds an acceptable fit of $\chi^{2}=10.2$ for 12 degrees of freedom. Here $k T=0.42_{-0.13}^{+0.23} \mathrm{keV}$. The $0.4-6 \mathrm{keV}$ luminosities in the thermal and power-law components are $(2.8 \pm 1.3) \times 10^{42}$ and $(2.9 \pm 1.0) \times 10^{42} \mathrm{erg} \mathrm{s}^{-1}$, respectively. The $1 \mathrm{keV}$ flux density of the power-law component is $1.2 \pm 0.4 \mathrm{nJy}$. Alternatively, a model with two thermal components of solar abundances finds a good fit $\left(\chi^{2}=9.7\right.$ for 11 dof $)$ with values for $k T$ of $0.39_{-0.13}^{+0.23}$ and $3.8_{-2.1}^{+u} \mathrm{keV}$, where the upper limit of the hotter thermal is unconstrained (Figure 11). The $0.4-6 \mathrm{keV}$ luminosities are comparable in the two components, at $(2.7 \pm 1.3) \times 10^{42}$ and $(2.7 \pm 1.0) \times 10^{42} \mathrm{erg} \mathrm{s}^{-1}$, respectively. The cool component, which would be associated with unshocked ISM, has a temperature consistent with the galaxy atmospheres of isolated ellipticals hosting nearby low-power radio sources, such as NGC 315 (Worrall et al. 2003), although with a luminosity roughly a factor of ten higher than expected. Mixed AGN emission could be responsible for some of the excess luminosity, or this may be a case where central collapse has both elevated the $\mathrm{X}$-ray luminosity and triggered the young radio source. Based on the Rankine-Hugoniot conditions for a strong shock (see equations for example in Worrall \& Birkinshaw 2006), the hot component would be consistent with arising from about $8 \%$ of the ISM mass in about $2 \%$ of the volume that has been shocked by a Mach number of about 5 . While this would seem a credible model, the sensitivity of the data limits the ability to draw definitive conclusions. 
Table 2

Properties of X-Ray Detected CSS Radio Galaxies

\begin{tabular}{|c|c|c|c|c|c|c|c|c|}
\hline Sources & $z$ & Size (kpc) & $\log P_{1.4 \mathrm{GHz}}$ & $\log L_{\mathrm{X}-\text { ray }}$ & Counts & Extended? & Shock? & References \\
\hline B0810+077 & 0.1122 & 3.0 & 25.16 & 42.8 (a) & 119 & $\mathrm{Y}$ & $\mathrm{N}$ & (1) \\
\hline B $0942+355$ & 0.2076 & 5.1 & 25.25 & 42.9 (a) & 103 & $\mathrm{Y}$ & $\mathrm{N}$ & (1) \\
\hline $3 \mathrm{C} 237$ & 0.877 & 9.2 & 28.3 & 44.0 (b) & $\ldots$ & Y & $\mathrm{N}$ & (9) \\
\hline $3 C 268.3$ & 0.3717 & 6.9 & 27.07 & 44.5 (b) & 398 & $\mathrm{~N}$ & $\mathrm{~N}$ & (3), (7) \\
\hline B $1321+045$ & 0.263 & 12 & 25.43 & 42.3 (b) & 53 & Y & $\mathrm{N}$ & (1), (2) \\
\hline $3 \mathrm{C} 303.1$ & 0.2704 & 7.0 & 26.63 & 42.9 (b) & 211 & Y & $\mathrm{Y}$ & (4), (5) \\
\hline B $1558+536$ & 0.179 & 6.0 & 25.19 & 41.7 (a) & 9 & $\mathrm{~N}$ & $\mathrm{~N}$ & (1) \\
\hline
\end{tabular}

Notes. Selected properties of CSS sources detected in X-rays. Column (1): source name. Column (2): redshift. Column (3): largest linear size (e.g., diameter) of the radio source in kpc. (a) Based on our new spectroscopic redshift, the candidate CSS source PKS B1017-325 is now known to have a projected linear diameter of $44 \mathrm{kpc}$, which is significantly above the cutoff for CSS sources. Thus, PKS B1017-325 is not considered a CSS for the analysis in Section 2.7. Column (4): log radio power at rest frame $1.4 \mathrm{GHz}\left(\mathrm{W} \mathrm{Hz}^{-1}\right)$. A radio spectral index of $\alpha=-0.75$ was adopted. Column (5): X-ray luminosity (erg s ${ }^{-1}$ ). (a) $2-10 \mathrm{keV}$, (b) $0.5-7 \mathrm{keV}$, and (c) 0.4-6 keV. Column (6): Chandra X-ray counts, except for 3C303.1 and PKS B1017-325, which are XMM-Newton counts. We use the same energy band as used in columns (5) and (7). Is there evidence for extended X-ray emission? (a) The constraint on PKS B1017-325 is based on XMM data which does not have sufficient angular resolution to rule out a galaxy-scale ISM. Column (8): is there evidence for shocked hot gas in the X-rays? Column (9): references for columns (5)-(8): (1) Kunert-Bajraszewska et al. (2014); (2) Kunert-Bajraszewska et al. (2013); (3) this paper; (4) O’Dea et al. (2006); (5) Massaro et al. (2010); 6. Massaro et al. (2009); (7) Massaro et al. (2015); (8) Hardcastle et al. (2012); (9) Massaro et al. (2017).
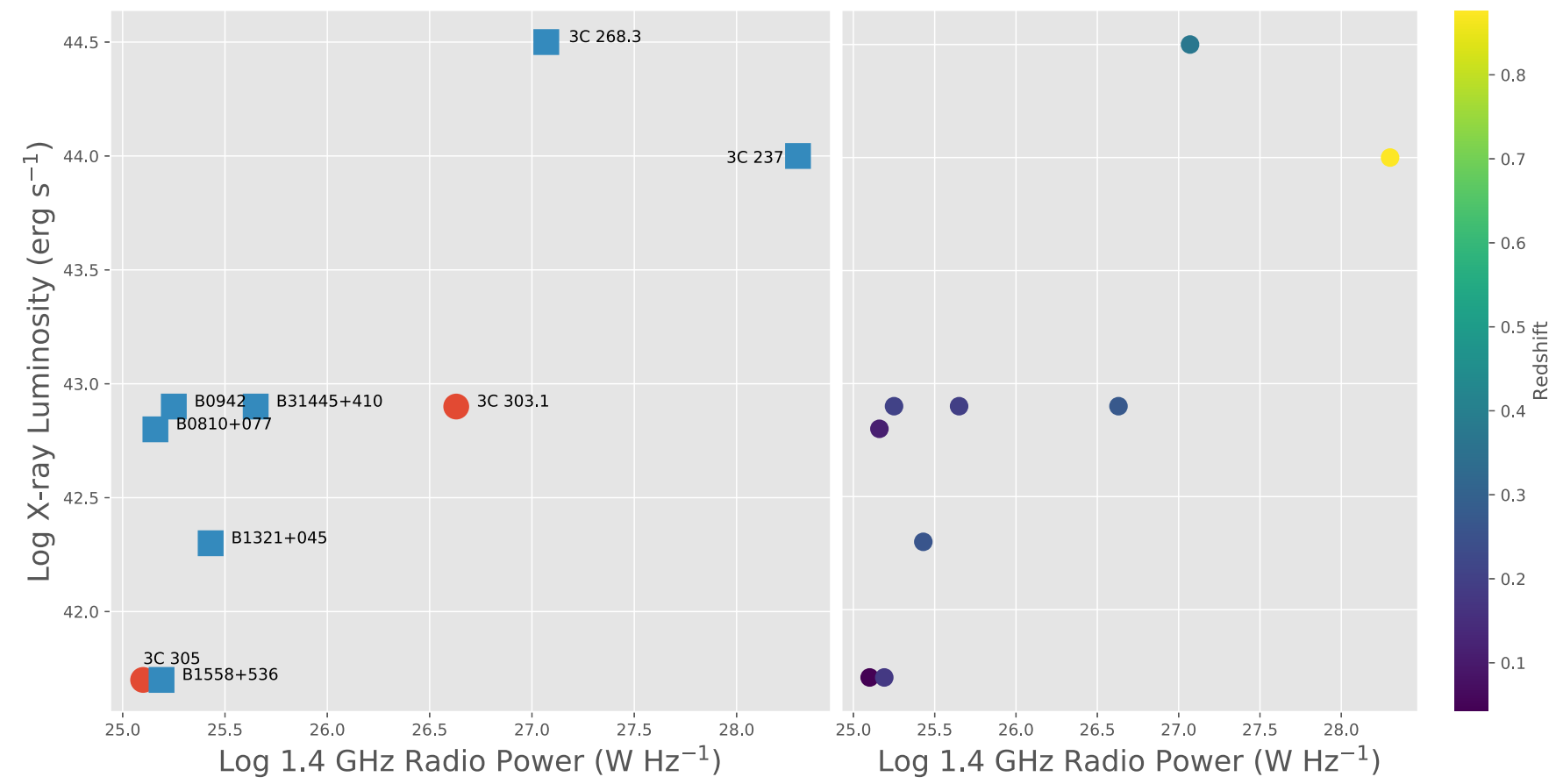

Figure 12. Log X-ray luminosity vs. Log $1.4 \mathrm{GHz}$ radio power for the nine CSS sources in Table 2. (Left) The sources with no X-ray spectral evidence for hot shocked gas are plotted as blue squares, and those with evidence for hot shocked gas are plotted as red circles. (Right) Same data, but color coded for redshift using the scale on the right.

\subsection{Comparison with other CSS Sources}

In Table 2 we present selected properties of the nine known CSS galaxies with detections in the X-ray. ${ }^{15}$ The counts are generally small. Even so, there is X-ray spectroscopic evidence for hot shocked gas in two of the nine CSS

15 Based on our new spectroscopic redshift, the candidate CSS source PKS B1017-325 is now known to have a projected linear diameter of $44 \mathrm{kpc}$, which is significantly above the cutoff for CSS sources. Thus, PKS B1017-325 is not considered a CSS for the analysis in this section.
galaxies-3C303.1 (O'Dea et al. 2006) and 3C305 (Massaro et al. 2009; Hardcastle et al. 2012). In addition, three of the CSS sources show X-ray emission elongated along the radio source-3C237 (Massaro et al. 2017), 3C303.1 (Massaro et al. 2010), and 3C305 (Massaro et al. 2009; Hardcastle et al. 2012). Figure 12 shows the X-ray luminosity versus radio power for the nine CSS sources with $\mathrm{X}$-ray detections (data taken from Table 2). We see an increase in X-ray luminosity with radio power as found by Kunert-Bajraszewska et al. (2014). The Pearson coefficient for this correlation is 0.802 , and the 
associated $p$-value is 0.009 . A partial correlation analysis between the X-ray and radio luminosity, controlling for redshift as a third variable, reveals that the two luminosities remain positively correlated. Redshift is nevertheless also a driver of the correlation (Figure 12), and, as with any luminosity versus luminosity plot (subject to Malmquist and "bigger is bigger" biases), caution should be used in the interpretation of this result.

The CSS radio galaxies with evidence for hot shocked gas are not exceptional in their radio or X-ray luminosities compared to the total sample of nine. We speculate that hot shocked gas is typical in CSS radio galaxies and that deeper $\mathrm{X}$-ray observations will reveal it.

\section{Conclusions}

We present X-ray, radio, and optical observations of three radio galaxies. 3C 268.3 is a broad-line CSS radio galaxy. B3 $1445+410$ is an LEG CSS radio galaxy with possibly a hybrid FRI/II (or fat double) radio morphology. The Chandra observations are point-like and well fit with a power law consistent with emission from a Doppler boosted core. The Chandra observations of 3C 268.3 are consistent with a point source centered on the nucleus whose spectrum can be fit by two power-law components. PKS B1017-325 is a lowexcitation emission line radio galaxy with a bent double radio morphology. The XMM-Newton observations are consistent with ISM emission with either a contribution from hot shocked gas or non-thermal jet emission. Using our new spectroscopic redshift, the projected linear size for PKS B1017-325 falls outside of the formal definition of a CSS, and thus, we drop PKS B1017-325 from consideration as a CSS source. We compile selected radio and X-ray properties of the nine bona fide CSS radio galaxies with X-ray detections so far. We find that two out of nine show X-ray spectroscopic evidence for hot shocked gas and three CSS sources show X-ray emission aligned with the radio source. We note that the counts in the sources are low and that the properties of the two sources with evidence for hot shocked gas are typical of the other CSS radio galaxies. We suggest that hot shocked gas may be typical of CSS radio galaxies due to their propagation through their host galaxies.

Facilities: CSO, LBT, VLA, XMM.

This work was partially supported by NASA grants NNX08AX40G and GO0-11125X. G.R.T. acknowledges support from NASA through Einstein Postdoctoral Fellowship Award Number PF-150128, issued by the Chandra X-Ray Observatory Center, which is operated by the Smithsonian Astrophysical Observatory for and on behalf of NASA under contract NAS8-03060. Basic research in radio astronomy at the Naval Research Laboratory is supported by 6.1 Base Funding. The National Radio Astronomy Observatory (NRAO) is operated by Associated Universities Inc. under cooperative agreement with the National Science Foundation.

This work also contains data obtained at the LBT, for which we gratefully acknowledge LBT Director Dr. Christian Veillet. The LBT is an international collaboration among institutions in the United States, Italy, and Germany. LBT Corporation partners are: The University of Arizona on behalf of the Arizona university system; Istituto Nazionale di Astrofisica, Italy; LBT Beteiligungsgesellschaft, Germany, representing the Max-Planck Society, the Astrophysical Institute Potsdam, and Heidelberg University; The Ohio State University, and The
Research Corporation, on behalf of The University of Notre Dame, University of Minnesota, and University of Virginia.

We also use data from the SDSS. Funding for the SDSS and SDSS-II has been provided by the Alfred P. Sloan Foundation, the Participating Institutions, the National Science Foundation, the U.S. Department of Energy, the National Aeronautics and Space Administration, the Japanese Monbukagakusho, the Max-Planck Society, and the Higher Education Funding Council for England. The SDSS Web Site is http://www. sdss.org/

This publication makes use of data products from the Two Micron All Sky Survey, which is a joint project of the University of Massachusetts and the Infrared Processing and Analysis Center/California Institute of Technology, funded by the National Aeronautics and Space Administration and the National Science Foundation.

\section{ORCID iDs}

D. M. Worrall (i) https://orcid.org/0000-0002-1516-0336

G. R. Tremblay (i) https://orcid.org/0000-0002-5445-5401

T. E. Clarke (iD https://orcid.org/0000-0001-6812-7938

B. Rothberg (i) https://orcid.org/0000-0003-2283-2185

\section{References}

An, T., \& Baan, W. A. 2012, ApJ, 760, 77

Axon, D. J., Capetti, A., Fanti, R., et al. 2000, AJ, 120, 2284

Birzan, L., Rafferty, D. A., McNamara, B. R., Wise, M. W., \& Nulsen, P. E. J. 2004, ApJ, 607, 800

Blanton, E. L., Gregg, M. D., Helfand, D. J., Becker, R. H., \& Leighly, K. M. 2001, AJ, 121, 2915

Boehringer, H., Voges, W., Fabian, A. C., Edge, A. C., \& Neumann, D. M. 1993, MNRAS, 264, L25

Burgess, A. M., \& Hunstead, R. W. 2006a, AJ, 131, 100

Burgess, A. M., \& Hunstead, R. W. 2006b, AJ, 131, 114

Buttiglione, S., Capetti, A., Celotti, A., et al. 2010, A\&A, 509, A6

Cardelli, J. A., Clayton, G. C., \& Mathis, J. S. 1989, ApJ, 345, 245

Carilli, C. L., Perley, R. A., \& Harris, D. E. 1994, MNRAS, 270, 173

Churazov, E., Gilfanov, M., Forman, W., \& Jones, C. 1996, ApJ, 471, 673

Cotton, W. D. 2008, PASP, 120, 439

Cotton, W. D., Dallacasa, D., Fanti, C., et al. 2003, PASA, 20, 12

Croston, J. H., Hardcastle, M. J., Mingo, B., et al. 2011, ApJL, 734, L28

Croston, J. H., Kraft, R. P., Hardcastle, M. J., et al. 2009, MNRAS, 395, 1999

de Vries, W. H., O’Dea, C. P., Baum, S. A., et al. 1997, ApJS, 110, 191

Dickey, J. M., \& Lockman, F. J. 1990, ARA\&A, 28, 215

Dunn, R. J. H., Fabian, A. C., \& Taylor, G. B. 2005, MNRAS, 364, 1343

Evans, D. A., Worrall, D. M., Hardcastle, M. J., Kraft, R. P., \& Birkinshaw, M. 2006, ApJ, 642, 96

Fabian, A. C. 2012, ARA\&A, 50, 455

Fanti, C., Fanti, R., Dallacasa, D., et al. 1995, A\&A, 302, 317

Fanti, C., Pozzi, F., Dallacasa, D., et al. 2001, A\&A, 369, 380

Fanti, R., Fanti, C., Schilizzi, R. T., et al. 1990, A\&A, 231, 333

Forman, W., Jones, C., Churazov, E., et al. 2007, ApJ, 665, 1057

Gallieni, D., Anaclerio, E., Lazzarini, P. G., et al. 2003, Proc. SPIE, 4839, 765

Guainazzi, M., Siemiginowska, A., Rodriguez-Pascual, P., \& Stanghellini, C. 2004, A\&A, 421, 461

Guainazzi, M., Siemiginowska, A., Stanghellini, C., et al. 2006, A\&A, 446, 87

Hardcastle, M. J., Evans, D. A., \& Croston, J. H. 2009, MNRAS, 396, 1929

Hardcastle, M. J., Massaro, F., Harris, D. E., et al. 2012, MNRAS, 424, 1774

Heckman, T. M., Miley, G. K., Balick, B., van Breugel, W. J. M., \& Butcher, H. R. 1982, ApJ, 262, 529

Heinz, S., Reynolds, C. S., \& Begelman, M. C. 1998, ApJ, 501, 126

Konami, S., Matsushita, K., Nagino, R., \& Tamagawa, T. 2014, ApJ, 783, 8

Kraft, R. P., Vázquez, S. E., Forman, W. R., et al. 2003, ApJ, 592, 129

Kunert-Bajraszewska, M., Labiano, A., Siemiginowska, A., \& Guainazzi, M. 2014, MNRAS, 437, 3063

Kunert-Bajraszewska, M., Siemiginowska, A., \& Labiano, A. 2013, ApJL, 772, L7

Leahy, J. P. 1991, in Beams and Jets in Astrophysics, ed. P. A. Hughes (Cambridge: Cambridge Univ. Press), 100 
Lüdke, E., Garrington, S. T., Spencer, R. E., et al. 1998, MNRAS, 299, 467

Massaro, F., Chiaberge, M., Grandi, P., et al. 2009, ApJL, 692, L123

Massaro, F., Harris, D. E., Liuzzo, E., et al. 2015, ApJS, 220, 5

Massaro, F., Harris, D. E., Liuzzo, E., et al. 2017, ApJS, in press

Massaro, F., Harris, D. E., Tremblay, G. R., et al. 2010, ApJ, 714, 589

Massaro, F., Harris, D. E., Tremblay, G. R., et al. 2013, ApJS, 206, 7

Massaro, F., Tremblay, G. R., Harris, D. E., et al. 2012, ApJS, 203, 31

Miller, D. L., Guerra, J. C., Boutsia, K., et al. 2012, Proc. SPIE, 8447, 84472 T

Morganti, R., Oosterloo, T. A., Tadhunter, C. N., van Moorsel, G., \& Emonts, B. 2005, A\&A, 439, 521

Murgia, M., Fanti, C., Fanti, R., et al. 1999, A\&A, 345, 769

O'Dea, C. P. 1998, PASP, 110, 493

O’Dea, C. P., De Vries, W. H., Worrall, D. M., Baum, S. A., \& Koekemoer, A. 2000, AJ, 119, 478

O'Dea, C. P., de Vries, W. H., Koekemoer, A. M., et al. 2002, AJ, 123, 2333

O’Dea, C. P., Mu, B., Worrall, D. M., et al. 2006, ApJ, 653, 1115

O’Dea, C. P., Worrall, D. M., Baum, S. A., \& Stanghellini, C. 1996, AJ, 111,92

Orienti, M. 2016, AN, 337, 9

Owen, F. N., \& Laing, R. A. 1989, MNRAS, 238, 357

Owen, F. N., \& Rudnick, L. 1976, ApJL, 205, L1

Owsianik, I., \& Conway, J. E. 1998, A\&A, 337, 69

Peck, A. B., \& Taylor, G. B. 2000, ApJ, 534, 90

Pogge, R. W., Atwood, B., Belville, S. R., et al. 2006, Proc. SPIE, 6269, 62690I

Polatidis, A. G. 2009, AN, 330, 149

Quirós-Pacheco, F., Busoni, L., Agapito, G., et al. 2010, Proc. SPIE, 7736, $77363 \mathrm{H}$

Rafferty, D. A., McNamara, B. R., Nulsen, P. E. J., \& Wise, M. W. 2006, ApJ, 652,216

Readhead, A. C. S., Taylor, G. B., Pearson, T. J., \& Wilkinson, P. N. 1996, ApJ, 460, 634

Reynaldi, V., \& Feinstein, C. 2013, MNRAS, 435, 1350

Rothberg, B., Kuhn, O., Edwards, M. L., et al. 2016, Proc. SPIE, 9906, 990622
Saikia, D. J., \& Gupta, N. 2003, A\&A, 405, 499

Scheuer, P. A. G. 1974, MNRAS, 166, 513

Schlafly, E. F., \& Finkbeiner, D. P. 2011, ApJ, 737, 103

Secrest, N. J., Satyapal, S., Gliozzi, M., et al. 2015, ApJ, 798, 38

Siemiginowska, A., Cheung, C. C., LaMassa, S., et al. 2005, ApJ, 632, 110

Siemiginowska, A., LaMassa, S., Aldcroft, T. L., Bechtold, J., \& Elvis, M. 2008, ApJ, 684, 811

Siemiginowska, A., Sobolewska, M., Migliori, G., et al. 2016, ApJ, 823, 57

Skrutskie, M. F., Cutri, R. M., Stiening, R., et al. 2006, AJ, 131, 1163

Smith, D. A., Wilson, A. S., Arnaud, K. A., Terashima, Y., \& Young, A. J. 2002, ApJ, 565, 195

Stockton, A., Canalizo, G., Fu, H., \& Keel, W. 2007, ApJ, 659, 195

Taylor, G. B., Marr, J. M., Pearson, T. J., \& Readhead, A. C. S. 2000, ApJ, 541,112

Tengstrand, O., Guainazzi, M., Siemiginowska, A., et al. 2009, A\&A, 501, 89

Tornikoski, M., Torniainen, I., Lähteenmäki, A., et al. 2009, AN, 330, 128

Tremblay, G. R., O'Dea, C. P., Baum, S. A., et al. 2010, ApJ, 715, 172

Tremblay, G. R., O’Dea, C. P., Baum, S. A., et al. 2012, MNRAS, 424, 1026

Vaiana, G. S., Cassinelli, J. P., Fabbiano, G., et al. 1981, ApJ, 245, 163

Vigotti, M., Grueff, G., Perley, R., Clark, B. G., \& Bridle, A. H. 1989, AJ, 98,419

Vink, J., Snellen, I., Mack, K.-H., \& Schilizzi, R. 2006, MNRAS, 367, 928

Watson, M. G., Schröder, A. C., Fyfe, D., et al. 2009, A\&A, 493, 339

Wise, M. W., McNamara, B. R., Nulsen, P. E. J., Houck, J. C., \& David, L. P. 2007, ApJ, 659, 1153

Worrall, D. M. 2009, A\&ARv, 17, 1

Worrall, D. M., \& Birkinshaw, M. 1994, ApJ, 427, 134

Worrall, D. M., \& Birkinshaw, M. 2006, in Physics of Active Galactic Nuclei at all Scales, ed. D. Alloin (Berlin: Springer), 39

Worrall, D. M., Birkinshaw, M., \& Hardcastle, M. J. 2003, MNRAS, 343, L73

Worrall, D. M., Birkinshaw, M., Young, A. J., et al. 2012, MNRAS, 424 1346

Worrall, D. M., Hardcastle, M. J., Pearson, T. J., \& Readhead, A. C. S. 2004 MNRAS, 347, 632 